\title{
Effects of processing bias on the recognition of composite face halves
}

\author{
NICOLA J. WESTON and TIMOTHY J. PERFECT \\ University of Plymouth, Plymouth, England
}

\begin{abstract}
Research has shown that face recognition accuracy can be improved by prior global processing and impaired by prior local processing (Macrae \& Lewis, 2002). The aim of this study was to test the processing bias account of face recognition, using the composite face task (Young, Hellawell, \& Hay, 1987), a test of featural recognition. Undergraduate volunteers $(N=75)$ participated in a between-subjects design that tested their ability to recognize face halves within a composite, following either global or local Navon processing or a control task. Results showed that, as compared with the control task, local processing speeded ability to recognize face halves. These results provide support for the processing bias account of face recognition.
\end{abstract}

Research has shown that face recognition is based on two types of processing: featural and configural (Diamond \& Carey, 1986; Leder \& Bruce, 2000; Tanaka \& Farah, 1993). Many tasks have manipulated both types of information to determine their importance in face recognition accuracy - for example, by comparing the recognition of isolated facial features versus whole faces (Tanaka \& Farah, 1993) or the recognition of spatial versus featural changes (Leder \& Bruce, 2000) and by investigating the effects of inversion (Bartlett \& Searcy, 1993; Thompson, 1980; Yin, 1969). It is clear that accurate face recognition is strongly influenced by orientation, with recognition hampered by inverted presentation (e.g., Bartlett \& Searcy, 1993; Thompson, 1980; Yin, 1969). Moreover, human faces have been found to be more vulnerable to stimulus inversion than is any other class of stimuli, suggesting that faces are special (Yin, 1969) or involve expert processing (Diamond \& Carey, 1986).

Additional evidence for holistic processing of faces has come from work on facial composites (Young, Hellawell, $\&$ Hay, 1987). In their task, Young et al. constructed facial composites by combining the top half of one famous face with the bottom half of another. Halves were presented either aligned, with the top half placed directly on top of the bottom half, or misaligned, with the top half placed off center with respect to the bottom half. Young et al. found that reaction times for naming composite face halves were significantly longer when the halves were aligned, as compared with when they were misaligned. They claimed that aligning the two halves created the illusion of a new face, which elicited holistic processing and, thus, interfered with identification, relative to the misaligned halves, which did not evoke this holistic-processing component.

This research was supported by sponsorship from the Economic and Social Research Council. Correspondence concerning this article should be addressed to N. J. Weston, School of Psychology, University of Plymouth, Plymouth PL4 8AA, England (e-mail: nicola.weston@plymouth.ac.uk).
Whereas earlier research was concerned with demonstrating that holistic information is involved in face recognition, more recent research has investigated the possibility of biasing processing style prior to a face recognition task. Macrae and Lewis (2002) tested the effect of prior global and local processing, induced by Navon (1977) stimuli, using an eyewitness paradigm. Participants viewed a video of a bank robbery and, following this, either acted as a control or were instructed to perform a letter identification task (Navon, 1977) for $10 \mathrm{~min}$. The stimuli in this task were large letters made up of several smaller mismatching letters (e.g., a T composed of Hs). The task was to report the identity of either the large letter (global processing) or the small letter (local processing). The participants were then asked to identify the person shown in the video from a simultaneous target-present lineup. Prior global processing led to better recognition performance (83\%) than did the control task $(60 \%)$, whereas prior local processing led to poorer recognition $(30 \%)$. These results, which have since been replicated (Perfect, 2003), are striking because they move beyond the straightforward demonstration that face processing involves a holistic component to the demonstration that processing modes can be induced and can impact upon subsequent face recognition accuracy.

However, Macrae and Lewis's (2002) argument that prior global processing of Navon (1977) stimuli improves face recognition through increased use of holistic processing of faces at test is open to question, because a lineup provides no measure of processing. As a recognition task, a lineup is not as straightforward as laboratory tasks involving the presentation of single faces; witnesses are invited to select a single face from a set of eight similar faces or to reject the entire set. Eyewitness research has shown there are a number of cognitive strategies witnesses may employ to make such a judgment (e.g., Dunning \& Stern, 1994), and to attribute increased success to increased holistic processing is entirely circular. Given that the Navon task had global precedence, any number of differences 
between conditions may be possible, such as differential difficulty of the Navon tasks as filler tasks or differential levels of arousal or motivation induced by the tasks.

What is required is a face recognition task with clear processing requirements. In the present study, the facial composite task (Young et al., 1987) was used. This task requires identification of composite face halves, which means that in order to make an accurate identification, participants have to overcome the misleading holistic information present when the halves are aligned. Consequently, using the task leads to a novel prediction: Engaging in prior local processing should aid recognition of composite halves, and global processing should hinder it. Such a reverse prediction is particularly powerful, since if supported, it would rule out any account of Macrae and Lewis's (2002) findings based on difficulty, motivation, or arousal. Such a local superiority effect has never been explored in the literature to date. On the basis of previous research showing that face-half recognition is not hampered when halves are misaligned, we predict that processing style will have a smaller effect on these stimuli, since they already evoke the relevant featural processing strategy needed for accurate recognition.

Because there has been little research on processing bias effects in face recognition, the longevity of any putative processing bias effect has yet to be explored. Until now, the effects of processing style have been explored only using a one-judgment face recognition task (Macrae \& Lewis, 2002; Perfect, 2003). To date, the only relevant evidence has come from the verbal overshadowing literature. The standard effect is that verbal description causes an impairment in subsequent lineup identification (e.g., Dodson, Johnson, \& Schooler, 1997; Fallshore \& Schooler, 1995; Schooler \& Engstler-Schooler, 1990; Westerman \& Larsen, 1997), which has been attributed to a processing bias that transfers from the description to the identification test (e.g., Dodson et al., 1997; Schooler, 2002). This effect has been tested using multiple trials, with mixed results (e.g., Brown \& Lloyd-Jones, 2002; Fallshore \& Schooler, 1995; Melcher \& Schooler, 1996; Ryan \& Schooler, 1998). Interestingly, only one of these studies has demonstrated a detrimental effect of verbal description over a series of trials (Brown \& Lloyd-Jones,
2002), with the remainder showing a verbal overshadowing effect only for the first trial following a verbal description. Given that trial effects have been found in the verbal overshadowing literature and the theoretical account of this effect is the same as that suggested for prior local processing of Navon stimuli, there is a strong possibility that trial effects will be present in this study. For this reason, the present study explored the magnitude of any processing bias effects across multiple trials following the processing bias interventions.

\section{METHOD}

\section{Participants}

Seventy-five members of the University of Plymouth community ( 17 of them male, 58 female) took part in this experiment for either payment or part of course credit.

\section{Stimuli}

The encoding and test face stimuli were color photographs of 52 males taken from the University of Stirling Psychology Department Psychological Image Collection (pics.psych.stir.ac.uk). All shoulders were removed from the photographs, and the background of each face was set to white. To create the facial composites, photos were divided by drawing a horizontal line across the bridge of the nose on each face (see Figure 1 for an example). The face stimuli were presented within a surface area $6 \mathrm{~cm}$ wide $\times 7.6 \mathrm{~cm}$ high. The experiment was programmed in Visual Basic 6 and run on a PC.

\section{Design and Procedure}

The experiment used a 2 (alignment) $\times 4$ (trial) $\times 3$ (processing bias) mixed design, with alignment and trial as within-subjects factors and processing bias manipulated between subjects.

The participants were tested individually and were randomly assigned to experimental conditions. The design comprised three phases; encoding, processing manipulation, and eight test trials. The participants completed all phases three times, making three test blocks.

At encoding, four intact faces were presented simultaneously on the screen, as a horizontal array, for $8 \mathrm{sec}$. The participants were instructed to remember the faces. During Phase 2, the global- and local-processing groups were instructed to complete the global and local versions of the Navon (1977) letter task. The participants were presented with 36 different mismatching Navon letters (e.g., an A composed of Bs) via a PowerPoint show lasting $3 \mathrm{~min}$. Each large letter was presented in font size 96, which was made up of smaller letters in Arial Black font type, font size 14. Each letter was displayed for $5 \mathrm{sec}$. Within this time, the participants had to say aloud

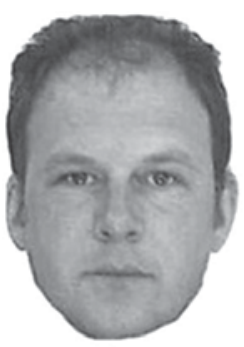

(A)

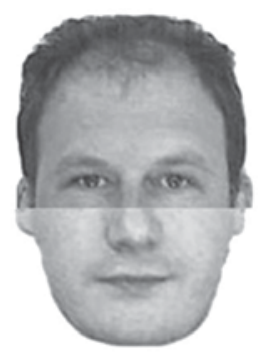

(B)

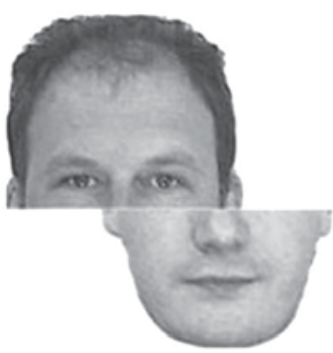

(C)

Figure 1. Examples of facial stimuli used in the experiment: (A) an original face, (B) an aligned facial composite, and (C) a misaligned facial composite. 
either the large letter (global processing) or the small letter (local processing). The control group was asked to complete a maze puzzle for $3 \mathrm{~min}$.

In Phase 3, the participants were given eight test trials in which they were asked to identify the face half that they had seen before. On each trial, there were two composites (Face A and Face B); one composite was made up of one half from the test stimulus (old half) and one other distractor half. The other composite was made up of two distractor halves. The position (i.e., in Face A or Face B) of the old half was randomized. The alignment of the stimulus was also randomized; on half of the trials, face halves were presented aligned, and on the other half, they were misaligned. Each test trial presented the participants with a question, which was either "Whose eyes have you seen before?" or "Whose mouth have you seen before?" The question was determined by the position of the old face half. If the old half was in the top half, the question would read "Whose eyes have you seen before?" On four trials, the old half was a bottom half, and on four trials, it was a top half. The two composites remained on the screen until the participants made a choice and continued until all eight trials had been completed.

These three phases were then repeated for the next block. For each block, a new set of four faces was used at encoding. The test faces and the distractor faces from the previous trials did not reappear in subsequent trials.

Prior to beginning the experiment, the participants took part in a practice trial in which they were presented with one face at encoding, the processing manipulation, and one test trial. Data from this were not analyzed.

\section{RESULTS AND DISCUSSION}

Accuracy and latency data were analyzed using threeway repeated measures ANOVAs. None of the variables interacted significantly with block; therefore, the data were collapsed across the three blocks. In order to examine the duration of the processing bias manipulation, the data were analyzed across the eight trials. Due to the small number of trials and to increase power, the data were split into 4 two-trial pairs. Thus, the first trial contained Trials 1 and 2 , the second trial contained Trials 3 and 4 , the third Trials 5 and 6, and the fourth Trials 7 and 8 .

The accuracy data did not yield any significant main effects of alignment $(F<1)$, processing condition $\left[F(2,60)=1.87, M S_{\mathrm{e}}=0.08, p=.16\right]$, or trial $(F<1)$. There were no significant two- or three-way interactions between these factors ( $F<1$ in all cases).

Analysis of mean response latencies showed a main effect of trial $\left[F(3,186)=15.33, M S_{\mathrm{e}}=579,672, p<.001\right.$; $\eta=.20]$ but no main effects of alignment $(F<1)$ or processing condition $\left[F(2,62)=2.03, M S_{\mathrm{e}}=5,955,679, p=\right.$ .14]. There was a significant interaction between trial and processing condition $\left[F(6,186)=2.34, M S_{\mathrm{e}}=579,672\right.$, $p<.05 ; \eta=.07]$. Results also showed a significant threeway interaction between alignment, trial, and processing condition $\left[F(6,186)=3.10, M S_{\mathrm{e}}=605,083, p<.01\right.$; $\eta=.09]$. The two-way interactions between alignment and trial $\left[F(3,186)=1.75, M S_{\mathrm{e}}=605,083, p=.16\right]$ and between alignment and processing condition $(F<1)$ were not significant.

Because the theoretical prediction was that processing bias would have a greater effect on aligned composites, separate analyses were carried out on the aligned and the misaligned composites. The top panel of Figure 2 shows the reaction times for each processing condition across trials for the aligned faces. A two-way repeated measures ANOVA showed a significant main effect of trial $\left[F(3,207)=11.33, M S_{\mathrm{e}}=603,867, p<.001 ; \eta=.14\right]$, showing decreased response latencies as trials progressed. The processing condition differences approached significance $\left[F(2,69)=2.59, M S_{\mathrm{e}}=3,805,849, p=.08\right]$, which was modified by a significant trial $\times$ processing condition interaction $\left[F(6,207)=2.96, M S_{\mathrm{e}}=603,867, p<\right.$ $.01 ; \eta=.08]$. Further analysis indicated that response latencies between conditions differed significantly over the first two trials after the manipulation [Trial $1, F(2,73)=$ $4.14, p<.05$; Trial 2, $F(2,74)=4.06, p<.05$ ], with quicker responses following local processing. For Trials 3 and 4, these condition differences had disappeared $(F \mathrm{~s}<1)$.

The bottom panel of Figure 2 shows response latencies across trials for the misaligned composites. Analysis from a two-factor repeated measures ANOVA showed a significant main effect of trial $\left[F(3,195)=7.40, M S_{\mathrm{e}}=610,713\right.$, $p<.001 ; \eta=.10]$, with decreased response latencies as trials progressed. There was no main effect of processing condition $\left[F(2,65)=1.20, M S_{\mathrm{e}}=3,597,728, p=\right.$
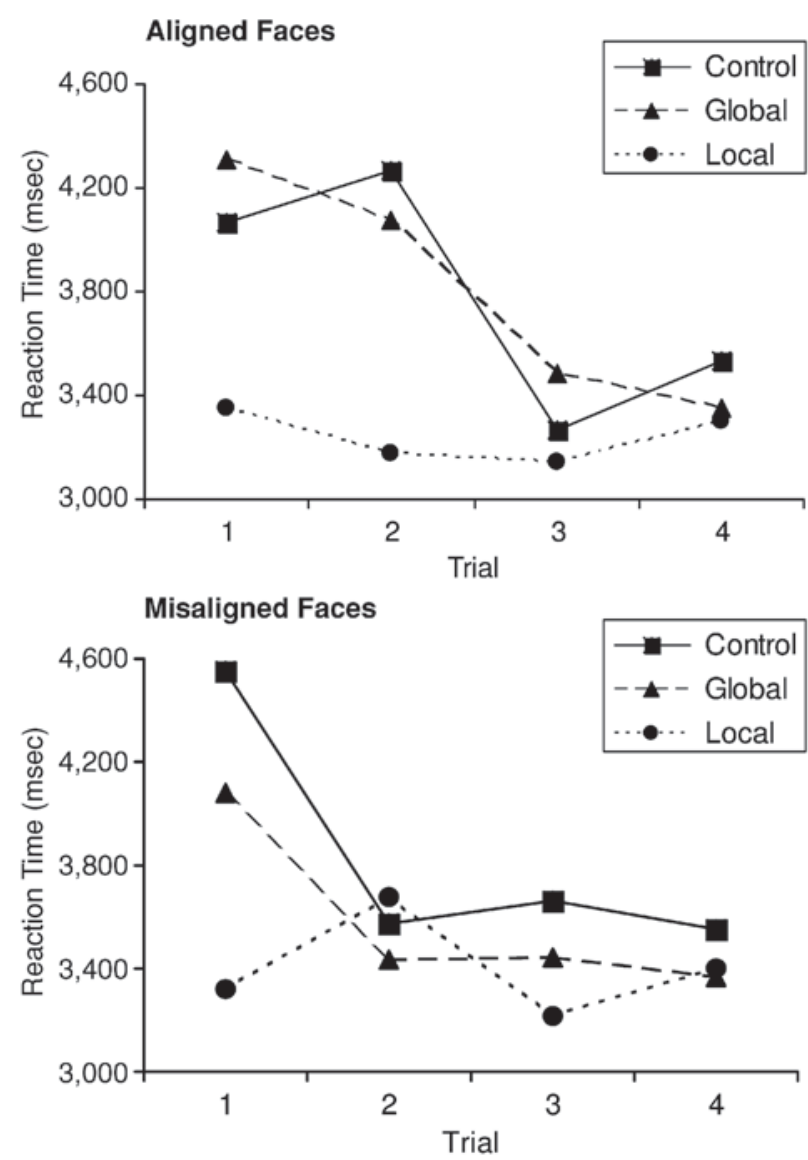

Figure 2. Mean reaction times for aligned (top panel) and misaligned (bottom panel) faces for each condition across trials. 
.31]. There was a significant trial $\times$ processing condition interaction $\left[F(6,195)=3.35, M S_{\mathrm{e}}=610,713, p<.01\right.$; $\eta=.09]$. Although there was a marginal local-processing advantage on the first trial, this advantage dissipated at a much greater rate over subsequent trials, as compared with the trials for the aligned halves. This result is not surprising, since the misaligned halves do not contain the same holistic component as the aligned halves and, thus, the advantage of local processing over control will be more modest for these stimuli.

These findings illustrate two important points. First, engaging in local processing decreases the time it takes to recognize composite halves when aligned composites are presented. Second, this advantage of prior local processing decreases as more judgments are made. We will discuss each of these findings in turn.

\section{Improvement Following Local Processing}

We argued earlier that the previous demonstrations of processing bias effects in face recognition, although interesting, could not be unambiguously attributed to differential use of featural and configural processing at test, because the test provided no independent measure of processing. Instead, it was possible that some other aspect of the Navon (1977) processing bias tasks could account for the changes in subsequent face recognition ability. However, the fact that we were able to show an opposite effect - quicker recognition performance following local, rather than global, processing in the facial composite task, in circumstances in which such an effect would be predicted - rules out these alternate explanations. If global processing of Navon stimuli somehow has an arousing or motivating effect, it should have aided this feature-based task, but it did not. This first demonstration of a local superiority effect, therefore, is a powerful confirmation of the theoretical account of processing bias effects and, at the same time, provides evidence for the generality of such processing bias effects across changes in encoding and test format. Previous demonstrations of processing bias effects occurred with videotaped presentation of a single target and tested memory with an eight-person simultaneous target-present lineup. Here, we obtained processing bias effects with the simultaneous presentation of four photographs of target faces at encoding and with a two-alternative forced choice test format. Furthermore, we demonstrated effects using response latency measures, as opposed to accuracy measures. The failure to find an effect on accuracy in this task may have been due to the higher performance demonstrated in this study because of the two-alternative test format, as compared with eight alternatives in a lineup.

Although our results showed the expected advantage following local processing, we did not find the expected disadvantage following global processing. One explanation for this pattern of results may consist of the way in which we presented the target faces at encoding. Initially, the participants were presented with four faces simultaneously on the screen for only $8 \mathrm{sec}$. This presentation method may have encouraged the participants to engage in a holistic-encoding strategy, encoding only general properties about the face, and not more detailed featural information. Consequently, when asked to recognize face halves, the participants in the control condition may have been predisposed to use a holistic strategy. Against a background of high holistic processing use in the control group, any manipulation designed to increase holistic-processing use is unlikely to have had a detectable effect. Of course, we acknowledge that this account is entirely speculative and requires further testing.

\section{The Longevity of the Processing Bias Effect}

This study, in which multiple face stimuli were used at encoding and test, allowed us to explore the longevity of the processing bias effect, in contrast to the standard eyewitness paradigm, which requires only one judgment. The response latency data for the aligned composites demonstrated that the induced processing mode is sustained only for a relatively small number of judgments. This suggests that although it is possible to temporarily bias processing style to a mode that is not normally used, people will eventually revert back to a default mode of processing elicited by the task.

This dissipation of the processing effect may have implications in regard to simultaneous versus sequential lineup presentation (Lindsay \& Wells, 1985). In a simultaneous lineup, participants see all the faces at once and make a single decision. Both Macrae and Lewis (2002) and Perfect (2003) demonstrated processing bias effects with simultaneous lineups. However, in a sequential lineup, participants are presented with each face individually and are asked to make a judgment for each face. The lineup terminates once an identification is made or all the faces have been rejected. If the effects of an induced processing mode are limited to only a few judgments, as the present data indicate, this would suggest that the effects are unlikely to transfer well to sequential lineups. Recent work in our laboratory has confirmed this pattern (Perfect, 2004).

\section{Theoretical Explanations of the Effect}

These data confirm previous studies demonstrating that face recognition accuracy can be influenced by a prior processing bias in interaction with the task demands. However, although it is appealing to attribute these effects to global and local modes of processing, some further attention to what these processing modes might constitute is warranted.

Until now, the theoretical focus has been on global/ local bias. That is, it has been argued that attending to the global or local properties of Navon (1977) letters biases people to go on to process the equivalent properties of the faces. Although this account has superficial appeal, it relies upon an assumed correspondence between the global (or local) level for Navon letters and the global (or local) level for faces. But does this correspondence exist? Although there is evidence that making a global decision about one Navon stimulus speeds the ability to make a global judgment about the stimulus on the next trial, ir- 
respective of the size of the stimulus (Robertson, 1996), there has been no evidence as yet that such priming effects transfer across tasks.

Even if such evidence were available, it would require careful experimentation to rule out explanations alternative to processing mode. One potential alternative basis for correspondence between the Navon (1977) letter tasks and the face identification task is spatial frequency. Clearly, identifying local or global properties of Navon stimuli requires attention to different spatial frequencies (Shulman \& Wilson, 1987) because the features are necessarily smaller than configurations of those features. The same argument applies to making configural and featural judgments about faces (Boutet, Collin, \& Faubert, 2003). Thus, one potential explanation of the processing bias effects observed to date is not that global- or localprocessing bias is being transferred but, instead, that they are due to attention to a particular spatial frequency. With respect to the present data, successful performance on the composite task requires attention toward high-frequency information and away from low-frequency information, since the latter leads to the misleading composite effect. Thus, prior attention to the features of the Navon stimuli, the high-frequency component, and away from the configural properties, the low-frequency component, is exactly the right combination that witnesses should apply to the composite task.

\section{REFERENCES}

Bartlett, J. C., \& Searcy, J. (1993). Inversion and configuration of faces. Cognitive Psychology, 25, 281-316.

Boutet, I., Collin, C., \& Faubert, J. (2003). Configural face encoding and spatial frequency information. Perception \& Psychophysics, 65, 1078-1093.

Brown, C., \& LLOYD-Jones, T. J. (2002). Verbal overshadowing in a multiple face presentation paradigm: Effects of description instruction. Applied Cognitive Psychology, 16, 873-885.

DiAMOND, R., \& CAREY, S. (1986). Why faces are and are not special: An effect of expertise. Journal of Experimental Psychology: General, 115, 107-117.

Dodson, C. S., Johnson, M. K., \& Schooler, J. W. (1997). The verbal overshadowing effect: Why descriptions impair face recognition. Memory \& Cognition, 25 (2), 129-139.

Dunning, D., \& Stern, L. B. (1994). Distinguishing accurate from inaccurate eyewitness identifications via inquiries about decision processes. Journal of Personality \& Social Psychology, 67, 818-835.

FALlshore, M., \& Schooler, J. W. (1995). Verbal vulnerability of perceptual expertise. Journal of Experimental Psychology: Learning, Memory, \& Cognition, 21, 1608-1623.

LEDER, H., \& BRUCE, V. (2000). When inverted faces are recognized: The role of configural information in face recognition. Quarterly Journal of Experimental Psychology, 53A, 513-536.

LiNDSAY, R. C. L., \& Wells, G. L. (1985). Improving eyewitness identifications from line-ups: Simultaneous versus sequential line-up presentation. Journal of Applied Psychology, 70, 556-564.

Macrae, C. N., \& Lewis, H. L. (2002). Do I know you? Processing orientation and face recognition. Psychological Science, 13, 194-196.

Melcher, J. M., \& Schooler, J. W. (1996). The misremembrance of wines past: Verbal and perceptual expertise differentially mediate verbal overshadowing of taste memory. Journal of Memory \& Language, 35, 231-245.

NAVON, D. (1977). Forest before trees: The precedence of global features in visual perception. Cognitive Psychology, 9, 353-383.

Perfect, T. J. (2003). Local processing bias impairs line-up performance. Psychological Reports, 93, 393-394.

Perfect, T. J. (2004, September). The effects of local and global processing on line-up performance. Paper presented at the BPS Cognitive Section Annual Conference, Leeds.

RoBERTSON, L. C. (1996). Attentional persistence for features of hierarchical patterns. Journal of Experimental Psychology: General, 125, 227-249.

Ryan, R. S., \& Schooler, J. W. (1998). Whom do words hurt? Individual differences in susceptibility to verbal overshadowing. Applied Cognitive Psychology, 12, S105-S125.

SCHOOLER, J. W. (2002). Verbalization produces a transfer inappropriate processing shift. Applied Cognitive Psychology, 16, 989-998.

Schooler, J. W., \& Engstler-Schooler, T. Y. (1990). Verbal overshadowing of visual memories: Some things are better left unsaid. Cognitive Psychology, 22, 36-71.

Shulman, G. L., \& Wilson, J. (1987). Spatial frequency and selective attention to local and global information. Perception, 16, 89-101.

Tanaka, J. W., \& Farah, M. J. (1993). Parts and wholes in face recognition. Quarterly Journal of Experimental Psychology, 46A, 225-245.

Thompson, P. (1980). Margaret Thatcher: A new illusion. Perception, 9, 483-484

Westerman, D. L., \& LaRsen, J. D. (1997). Verbal overshadowing effect: Evidence for a general shift in processing. American Journal of Psychology, 110, 417-428.

YIN, R. K. (1969). Looking at upside-down faces. Journal of Experimental Psychology, 81, 141-145.

Young, A. W., Hellawell, D., \& Hay, D. C. (1987). Configural information in face perception. Perception, 16, 747-759.

(Manuscript received December 15, 2004; revision accepted for publication April 11, 2005.) 